But are we any closer to understanding what these repeat RNAs do? In both experimental flies and human premutation patients, nuclear aggregates are seen that also contain ubiquitin, and so it is possible that here the RNA repeats sequester vital proteins from their normal functions. The fact that the formation of these clumps can be reversed by overexpressing the chaperone heat shock protein 70 (Hsp70), which normally unravels or destroys badlyfolded proteins, is intriguing: the involvement of the protein-degradation machinery could link these RNA-mediated defects to the larger class of protein-based neurodegenerative disorders, many of which are also reversed by Hsp70.

The creation of a Drosophila model for this disease has already paved the way for targeted genetic studies. Meanwhile, it looks like fragile $\mathrm{X}$ is back where it started - in a class of its own.

Tanita Casci, Senior Editor, Nature Reviews Genetics

6) References and links

original RESEARCH PAPER Jin, P. etal. RNA-mediated

neurodegeneration caused by the fragile $X$ premutation $\mathrm{rCGG}$

repeats in Drosophila. Neuron 39, 739-747 (2003)

WEB SITE

Stephen Warren's laboratory:

http://www.emory.edu/WHSC/MED/GENETICS/visitors/ warren.html

invaginates from the roof plate. The choroid plexus is best known as a producer of cerebrospinal fluid, but it is also thought to have a dorsoventral patterning function in the hindbrain. The authors confirmed that the choroid plexus cells are descendents of the same Wnt1-expressing population as the roof plate, and that like the roof plate cells, they respect boundaries.

The restriction of roof plate and choroid plexus cells to lineage-restricted compartments with distinct gene expression profiles makes these structures strong candidates for providing signals for the generation of neuronal diversity along the anteroposterior axis. By analysing and manipulating the gene expression profiles of specific roof plate and choroid plexus compartments, it should be possible to define their anteroposterior patterning roles more precisely.

Heather Wood

(2) References and links ORIGINAL RESEARCH PAPER Awatramani, R. et al. Cryptic boundaries in roof plate and choroid plexus identified by intersectional gene activation. Nature Genet. 35, 70-75 (2003)

FURTHER READING Caspary, T. \& Anderson, K. V. Patterning cell types in the dorsal neural tube: what the mouse mutants say. Nature Rev. Neurosci. 4, 289-297 (2003) | Zinyk, D. et al. Fate mapping of the mouse midbrain-hindbrain constriction using a site-specific recombination system. Curr. Biol. 8, 665-668 (1998)

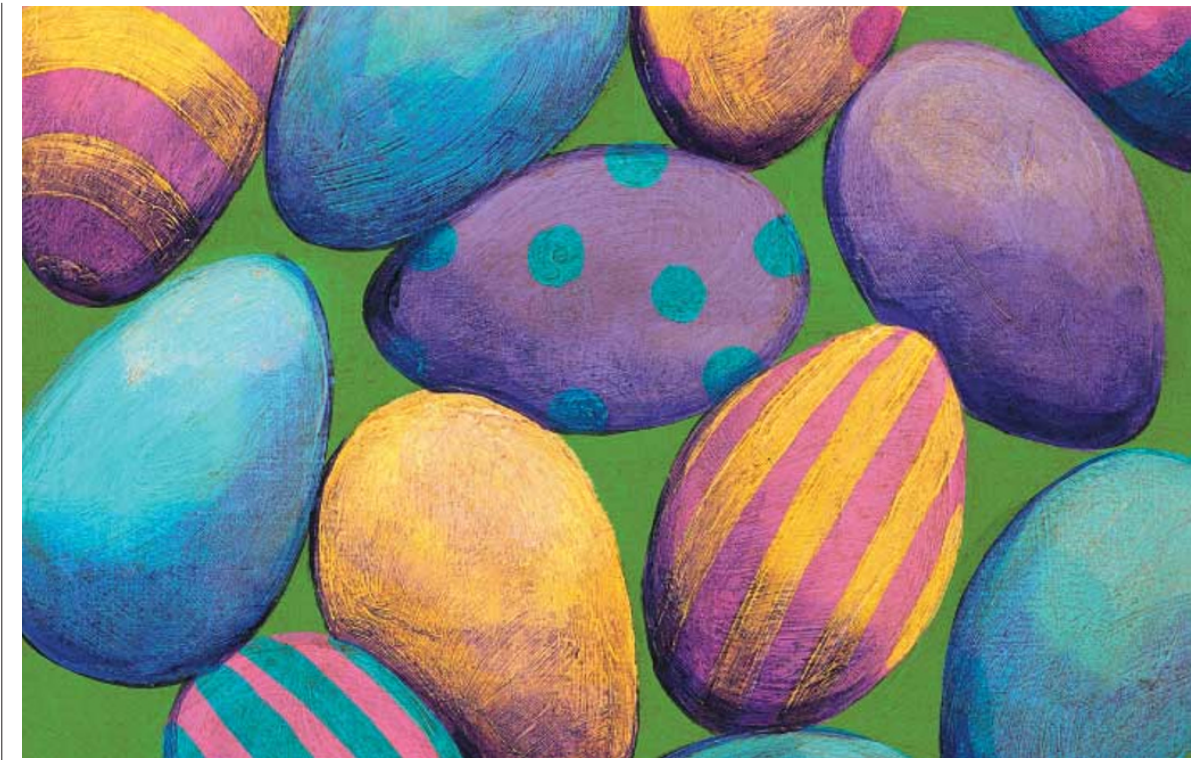

BEHAVIOURAL NEUROSCIENCE

\section{Decisions, decisions}

"You must learn to consider the consequences of your actions." How many times did we hear that while growing up? Being able to predict the outcome of an action - and to know whether that outcome is desirable - is essential for us to function normally. New evidence supports the idea that a prelimbic corticothalamic circuit is crucial for the ability of rats to make use of specific associations between actions and outcomes in an instrumental conditioning task.

Corbit et al. set out to distinguish the roles of two thalamic nuclei in this task. The mediodorsal nucleus is part of a circuit that includes the prelimbic part of the prefrontal cortex, whereas the anterior thalamic nucleus is linked to the hippocampus. Previous studies had suggested that mediodorsal lesions affected instrumental behaviour, but in some cases the lesions also affected the anterior nucleus. This, coupled with evidence that the hippocampal circuit, which includes the anterior nucleus, is important for some forms of learning, led to suggestions that impairments in action-outcome learning might have arisen from anterior damage, rather than from the mediodorsal lesion itself. The new data confirm that it is the mediodorsal thalamus, not the anterior nucleus, that is responsible.

Rats with anterior thalamic lesions performed normally on all measures tested. By contrast, rats with mediodorsal lesions showed specific impairments. Although they could learn to press a lever for food pellets and another for sucrose, they were less sensitive than control rats to manipulations of the outcome. For example, if trained rats are allowed to eat as much as they want before a test, they will normally press the lever that previously delivered food pellets less often than one that delivered the sucrose drink. Mediodorsal lesions reduced the sensitivity of the rats to this specific, satietyinduced devaluation of one reward.

In another test, the relationship between pressing one lever and delivery of its associated reward was altered by delivering one of the two rewards independently of lever pressing as well as when the lever was pressed. Unlesioned rats and rats with anterior thalamic lesions showed reduced leverpressing when it was no longer necessary to receive the reward, but rats with mediodorsal lesions continued to press both levers equally.

Control experiments showed that the mediodorsal lesions did not prevent the rats from distinguishing between the two rewards or the two levers. Rather, it seems that they impair the ability of the rats to learn and use information about the link between an action and its outcome. Prelimbic cortical lesions have similar effects, supporting the idea that a prelimbic-mediodorsal circuit supports action-outcome learning in this kind of task.

Rachel Jones

(1) References and links

ORIGINAL RESEARCH PAPER Corbit, L. H. et al. Lesions of mediodorsal thalamus and anterior thalamic nuclei produce dissociable effects on instrumental conditioning in rats. Eur. J. Neurosci. 18, 1286-1294 (2003) 